\title{
Association of Plasma B Lymphocyte Stimulator Levels and Disease Activity in Systemic Lupus Erythematosus
}

\author{
Michelle Petri, ${ }^{1}$ William Stohl, ${ }^{2}$ Winn Chatham, ${ }^{3}$ W. Joseph McCune, ${ }^{4}$ Marc Chevrier, ${ }^{5}$ \\ Jeff Ryel, ${ }^{5}$ Virginia Recta, ${ }^{5}$ John Zhong, ${ }^{5}$ and William Freimuth ${ }^{5}$
}

Objective. To determine the association of plasma B lymphocyte stimulator (BLyS) levels, immunosuppressive therapy, and other clinical parameters with disease activity in systemic lupus erythematosus (SLE).

Methods. Two hundred forty-five SLE patients were evaluated prospectively over a 2-year period at 4 centers. Assessments were performed every 3-6 months. Univariate analysis was used to determine the association among the Safety of Estrogens in Lupus Erythematosus: National Assessment (SELENA) version of the Systemic Lupus Erythematosus Disease Activity Index (SLEDAI) score, serum anti-double-stranded DNA (anti-dsDNA), and plasma BLyS levels. A multivariate repeated-measures model incorporating immunosuppressive therapy was utilized.

Results. Ninety-two percent of the patients were

Supported in part by Human Genome Sciences. The Hopkins Lupus Cohort is supported by the NIH (National Institute of Arthritis and Musculoskeletal and Skin Diseases grant R01-AR-43737) and by the Johns Hopkins Hospital Outpatient General Clinic Research Center (NIH grant M01-RR-00052).

Michelle Petri, MD, MPH: Johns Hopkins University School of Medicine, Baltimore, Maryland; ${ }^{2}$ William Stohl, MD, PhD: Los Angeles County + University of Southern California Medical Center, and University of Southern California Keck School of Medicine, Los Angeles; ${ }^{3}$ Winn Chatham, MD: University of Alabama at Birmingham; ${ }^{4} \mathrm{~W}$. Joseph McCune, MD, PhD: University of Michigan, Ann Arbor; ${ }^{5}$ Marc Chevrier, MD, PhD, FACR (current address: Centocor, Inc., Horsham, Pennsylvania), Jeff Ryel, RN, Virginia Recta, MS, John Zhong, PhD, William Freimuth, MD, PhD: Human Genome Sciences, Inc., Rockville, Maryland.

Dr. Petri has received consulting fees from Human Genome Sciences and GlaxoSmithKline (less than \$10,000 each). Dr. Stohl has received fees for Web-based lectures and grant support from Human Genome Sciences (less than $\$ 10,000$ each). Dr. Chatham has received honoraria from Human Genome Sciences (less than \$10,000). Drs. Zhong and Freimuth own stock or stock options in Human Genome Sciences.

Address correspondence and reprint requests to Michelle Petri, MD, MPH, Johns Hopkins University School of Medicine, Division of Rheumatology, 1830 East Monument Street, Suite 7500, Baltimore, MD 21205. E-mail: mpetri@jhmi.edu.

Submitted for publication March 5, 2007; accepted in revised form April 14, 2008. female. Sixty-seven percent were white, $31 \%$ African American, and 2\% Asian (all of these groups may include Hispanic). Mean values at baseline were as follows: age 41.5 years, disease duration 8.1 years, SELENA-SLEDAI 3.3 (median 2, range 0-18), BLyS $5.57 \mathrm{ng} / \mathrm{ml}$, IgG 1,439 mg/dl, C3 $104.4 \mathrm{mg} / \mathrm{dl}$, and C4 21.3 $\mathrm{mg} / \mathrm{dl}$; among those positive for anti-dsDNA, the median titer was 1:40 (range 1:10-1:1,280). Univariate analysis showed that plasma BLyS levels were associated with anti-dsDNA titers $(P=\mathbf{0 . 0 4 6 5})$ and SELENA-SLEDAI scores $(P=0.0002)$. In multivariate analyses, a greater increase in the SELENA-SLEDAI score from the previous visit was associated with higher BLyS levels at the previous visit $(P=0.0042)$ and with a greater increase in the BLyS level from the previous visit $(P=0.0007)$.

Conclusion. The findings of association between a greater increase in the BLyS level from the previous visit and a greater increase in the SELENA-SLEDAI score at the subsequent visit, and between an elevated BLyS level at the previous visit and a greater SELENASLEDAI score at the subsequent visit, demonstrate a relationship between circulating BLyS levels and SLE disease activity. These results lend support to the notion that BLyS is a candidate for therapeutic targeting in SLE.

B lymphocyte stimulator (BLyS; trademark of Human Genome Sciences, Rockville, MD), also known as BAFF (B cell-activating factor from the tumor necrosis factor [TNF] family), is a type II transmembrane protein (1). BLyS exists in both membrane-bound and soluble forms and is expressed on monocytes, macrophages, and monocyte-derived dendritic cells. BLyS gene expression and levels of membrane-associated soluble BLyS are regulated by cytokines, in particular interferon- $\gamma$ and to a lesser extent interleukin-10 (2). The biologically active soluble form of BLyS binds to 3 receptors expressed primarily on B lymphocytes: TACI (TNF transmembrane activator and calcium modulator 
and cyclophilin ligand interactor), BCMA (B lymphocyte maturation antigen), and BR3 (BAFF/BLyS receptor 3) $(3,4)$. Activation of the BLyS receptors leads to B cell and plasma cell proliferation, differentiation, and survival and IgG class switching $(1,5,6)$. A related TNF family member, APRIL (a proliferation-inducing ligand), can also bind TACI and BCMA and can mediate effects similar to those of BLyS, but its biologic action may be primarily on memory plasma cells. BLyS-APRIL heterotrimers have also been characterized (7), but their function in vivo is unclear.

Circulating BLyS levels are elevated in patients with systemic lupus erythematosus (SLE), rheumatoid arthritis (RA), and other systemic immune-based rheumatic diseases. A correlation between plasma BLyS protein levels and rheumatoid factor titers in patients with RA has been reported $(8,9)$. In addition, elevated levels of BLyS have been detected in the synovial fluid of patients with RA and have been correlated with levels of autoantibodies associated with Sjögren's syndrome (10). In cross-sectional studies, $20-40 \%$ of patients with SLE appear to have significantly higher levels of circulating BLyS protein than are found in normal controls, and plasma BLyS protein levels appear to correlate with IgG levels and anti-double-stranded DNA (anti-dsDNA) titers $(8,9)$. In SLE and RA patients treated with rituximab, BLyS levels increase with B cell depletion and return to near-baseline levels upon B cell repopulation $(11,12)$.

Although the findings of elevated levels of BLyS protein in autoimmune diseases suggest that BLyS may be a marker of current or impending autoimmune disease activity and may be a potential target for therapeutic intervention, the relationship between circulating BLyS levels and SLE disease activity has not been adequately characterized. Accordingly, this 2-year observational study was designed to assess the relationships between plasma BLyS concentrations and disease activity, autoantibody and immunoglobulin levels, and treatments received for SLE.

\section{PATIENTS AND METHODS}

Patients and study design. This 2-year multicenter observational study was undertaken to assess disease activity, therapies, and multiple biomarkers including plasma BLyS levels in patients with SLE as defined by the American College of Rheumatology (ACR) classification criteria $(13,14)$. Patients received standard-of-care therapy for their disease. To be eligible for the study, patients had to be at least 18 years old and could not have been treated with an investigational agent within 28 days prior to the start of the study. None of the study patients were found to have received an investigational agent at any time. Originally designed as a single-year natural history investigation, the study was extended to measure BLyS and evaluate disease activity through a second year. Patients were assessed clinically, and blood and urine samples were collected every 3 months during the first year of the study and every 6 months during the second year. Patients were also assessed during any unscheduled clinical visits that were necessary due to acute exacerbation of SLE disease activity. Written informed consent was obtained from all study patients prior to enrollment.

Patient assessment. At baseline, the medical history was reviewed and information on current medications and on the components of the ACR SLE classification criteria that the patient fulfilled was recorded. To assess disease activity, the Safety of Estrogens in Lupus Erythematosus: National Assessment (SELENA) version of the Systemic Lupus Erythematosus Disease Activity Index (SLEDAI) $(15,16)$ was administered, and plasma $\mathrm{BLyS}, \mathrm{C} 3 / \mathrm{C} 4$, and anti-dsDNA autoantibodies measured, at baseline, at 3, 6, 9, 12, 18, and 24 months, and at any unscheduled visits. Total immunoglobulins and subclasses (IgM, IgA, IgG, and $\operatorname{IgE}$ ) were measured at baseline and at the 6-, 12-, 18-, and 24-month scheduled visits. A complete blood cell count and urinalysis were performed at baseline, at 3, 6, 9, 12, 18, and 24 months, and at any unscheduled visit.

To measure plasma BLyS levels, $\sim 10 \mathrm{ml}$ of blood was collected in Vacutainer tubes containing EDTA, centrifuged for 15 minutes at 1,500 revolutions per minute, aliquoted $(0.5$ $\mathrm{ml}$ ) into cryotubes, and immediately frozen at $-70^{\circ} \mathrm{C}$. Plasma BLyS levels were measured by enzyme-linked immunosorbent assay (ELISA) with mouse anti-human BLyS monoclonal antibody 3D4 as the capture antibody and biotinylated rabbit anti-human BLyS polyclonal antibody as the detector antibody. Recombinant soluble BLyS was used to standardize the assay (interassay variability $<15 \%)(17)$. The normal plasma BLyS level measured using this assay ranged from $1 \mathrm{ng} / \mathrm{ml}$ to $5 \mathrm{ng} / \mathrm{ml}$ in healthy adults. The BLyS assay was performed at Human Genome Sciences Laboratory. Serum levels of biologic markers, including anti-dsDNA, total immunoglobulin, and immunoglobulin subclasses, were also measured by ELISA.

Statistical analysis. Disease history, use of immunosuppressive agents, and baseline demographic characteristics were compared among study sites (see below). These comparisons were performed using the likelihood ratio test for discrete variables and analysis of variance (ANOVA) or Wilcoxon's rank sum test, as appropriate, for continuous variables.

Descriptive statistics were used to summarize demographic and baseline disease characteristics, as well as changes in BLyS levels over time. Univariate association analysis was used to analyze BLyS levels and other variables, such as SELENA-SLEDAI scores and immunoglobulin levels. The significance of differences in BLyS levels between patients with and those without detectable anti-dsDNA antibodies was assessed by Student's $t$-test.

A repeated-measures analysis of covariance model was used to examine the relationship between disease activity and BLyS level, immunosuppressive drug use, and steroid dosage. In this model, the dependent variable was the change in SELENA-SLEDAI score from the previous to the current visit, and the explanatory variables were 1) BLyS level at the previous visit, 2) change in BLyS level from the previous to the 
Table 1. Demographic and baseline disease characteristics of the patients, by study site*

\begin{tabular}{|c|c|c|c|c|c|}
\hline & \multicolumn{4}{|c|}{ Clinical center } & \multirow[b]{2}{*}{$P \dagger$} \\
\hline & $\begin{array}{c}\text { JHU } \\
(n=100)\end{array}$ & $\begin{array}{c}\text { USC } \\
(\mathrm{n}=69)\end{array}$ & $\begin{array}{c}\text { UAB } \\
(\mathrm{n}=50)\end{array}$ & $\begin{array}{c}\text { UM } \\
(\mathrm{n}=26)\end{array}$ & \\
\hline Sex & & & & & 0.733 \\
\hline Female & $90(90)$ & $65(94)$ & $47(94)$ & $24(92)$ & \\
\hline Male & $10(10)$ & $4(6)$ & $3(6)$ & $2(8)$ & \\
\hline Race $\ddagger$ & & & & & $<0.001$ \\
\hline White & $51(51)$ & $62(90) \S$ & $27(54)$ & $23(88)$ & \\
\hline African American & $47(47)$ & $4(6)$ & $23(46)$ & $3(12)$ & \\
\hline Asian & $2(2)$ & $3(4)$ & 0 & 0 & \\
\hline Age, mean $\pm \mathrm{SD}$ years & $43 \pm 12$ & $41 \pm 13$ & $39 \pm 11$ & $43 \pm 15$ & 0.372 \\
\hline Duration of SLE, mean \pm SD years & $8.8 \pm 7.7$ & $8.7 \pm 9.3$ & $4.6 \pm 4.7$ & $11.0 \pm 9.7$ & 0.003 \\
\hline SELENA-SLEDAI score & & & & & $<0.001$ \\
\hline Median & 2.0 & 4.0 & 4.0 & 2.0 & \\
\hline Mean \pm SD & $2.0 \pm 2.4$ & $4.7 \pm 4.5$ & $4.3 \pm 3.6$ & $3.1 \pm 3.0$ & \\
\hline Prednisone use & $53(53)$ & $58(84)$ & $35(70)$ & $19(73)$ & 0.0002 \\
\hline Prednisone dosage, median (range) mg/day & $7.5(1-140)$ & $10(1-40)$ & $10(2.5-20)$ & $5(1-15)$ & 0.0200 \\
\hline \multicolumn{6}{|l|}{ Immunosuppressive drug use } \\
\hline Azathioprine & $7(7)$ & $36(52.2)$ & $14(28)$ & $2(7.7)$ & $<0.0001$ \\
\hline Mycophenolate mofetil & $15(15)$ & $3(4.3)$ & 0 & $12(46.2)$ & $<0.0001$ \\
\hline Cyclophosphamide & $13(13)$ & $4(5.8)$ & $2(4)$ & 0 & 0.1013 \\
\hline Methotrexate & $9(9)$ & $5(7.2)$ & $11(22)$ & $4(15.4)$ & 0.0787 \\
\hline Hydroxychloroquine & $63(63)$ & $59(86)$ & $37(74)$ & $19(73)$ & 0.0149 \\
\hline \multicolumn{6}{|l|}{ Components of SLE } \\
\hline ANA positive & $97(97)$ & $63(91)$ & $49(98)$ & $25(96)$ & 0.290 \\
\hline Arthritis & $62(62)$ & $50(72)$ & $41(82)$ & $21(81)$ & 0.039 \\
\hline Malar rash & $50(50)$ & $30(43)$ & $28(56)$ & $12(46)$ & 0.583 \\
\hline Photosensitivity & $48(48)$ & $30(43)$ & $24(48)$ & $14(54)$ & 0.830 \\
\hline Immunologic & $39(39)$ & $40(58)$ & $19(38)$ & $14(54)$ & 0.048 \\
\hline Hematologic & $49(49)$ & $30(43)$ & $16(32)$ & $11(42)$ & 0.261 \\
\hline Oral ulcers & $42(42)$ & $17(25)$ & $25(50)$ & $6(23)$ & 0.009 \\
\hline Renal & $28(28)$ & $31(45)$ & $11(22)$ & $9(35)$ & 0.040 \\
\hline Serositis & $26(26)$ & $19(28)$ & $8(16)$ & $5(19)$ & 0.404 \\
\hline Discoid rash & $18(18)$ & $5(7)$ & $5(10)$ & $5(19)$ & 0.137 \\
\hline Neurologic & $7(7)$ & $2(3)$ & 0 & $2(8)$ & 0.077 \\
\hline
\end{tabular}

* Except where indicated otherwise, values are the number $(\%)$. JHU = Johns Hopkins University; USC = University of Southern California; $\mathrm{UAB}=$ University of Alabama at Birmingham; UM = University of Michigan; SLE = systemic lupus erythematosus; SELENA-SLEDAI = Safety of Estrogens in Lupus Erythematosus: National Assessment version of the SLE Disease Activity Index; ANA = antinuclear antibody.

$\dagger$ Significance of the difference among sites, by analysis of variance, Wilcoxon's rank sum test, or likelihood ratio test.

\$ All race categories may include patients of Hispanic origin.

$\S$ Majority were Hispanic.

I One patient took cyclosporin A during the study. No patients took biologic agents.

current visit, 3) whether the patient was receiving prednisone in the last 3 months, 4) change in the prednisone dosage from the previous to the current visit, 5) whether the patient was receiving immunosuppressive treatment in the last 3 months, and 6) SELENA-SLEDAI score at the previous visit. The model was also adjusted for study site, the patient's race (white versus other), the interaction effect between study site and race, and age. A spatial covariance structure was assumed to account for the correlation between repeated observations within each patient.

\section{RESULTS}

Demographic and baseline clinical characteristics of the patients. A total of 245 patients at 4 clinical centers (Johns Hopkins University, University of South- ern California, University of Alabama at Birmingham, and University of Michigan) were enrolled in this observational study. Patient demographic characteristics, baseline disease characteristics, and ACR classification criteria by clinical center are summarized in Table 1 . Approximately $90 \%$ of the patients at each clinical center were female. The mean age was 41.5 years. The majority of the patients were white (ethnicity was selfreported, with $67 \%$ white, $31 \%$ African American, and $2 \%$ Asian [all of these ethnicity categories could include subjects of Hispanic origin]).

The mean duration of SLE was 8.1 years, and the mean baseline SELENA-SLEDAI score was 3.3 (median 2, range 0-18). The SLE study population was 
Table 2. Baseline levels of BLyS, immunoglobulin subgroups, and anti-dsDNA*

\begin{tabular}{lrrc}
\hline \multicolumn{1}{c}{ Parameter } & Mean & Median & Range \\
\hline BLyS, ng/ml (normal 1-5) & 5.57 & 4.85 & $1.5-9.1$ \\
Immunoglobulin subgroup $\dagger$ & & & \\
IgG, mg/dl (normal 694-1,618) & 1,439 & 1,325 & $461-5,180$ \\
IgA, mg/dl (normal 84-463) & 303 & 279 & $0-1,110$ \\
IgM, mg/dl (normal 48-291) & 132 & 116 & $3-708$ \\
Anti-dsDNA, titer (by IF) & NA & $1: 40$ & $1: 10-1: 1,280$ \\
$\quad$ (normal <1:30) $\$$ & & & \\
C3, mg/dl & 104.4 & 99 & $24.5-189$ \\
C4, mg/dl & 21.3 & 20 & $0-145$ \\
\hline
\end{tabular}

* Values are for patients from all study sites combined. BLyS $=\mathrm{B}$ lymphocyte stimulator; anti-dsDNA = anti-double-stranded DNA; $\mathrm{NA}=$ not appropriate.

$\dagger$ Normal ranges from Quest Diagnostics (Van Nuys, CA). Thirty-nine percent of the patients had IgG levels $>1,500 \mathrm{mg} / \mathrm{dl}, 16 \%$ had $\operatorname{IgA}$ levels $>460 \mathrm{mg} / \mathrm{dl}$, and $6 \%$ had IgM levels $>270 \mathrm{mg} / \mathrm{dl}$.

$\ddagger$ Only 60 patients $(24 \%)$ had detectable levels $(>1: 10)$ by immunofluorescence (IF) assay.

observed to be heterogeneous as indicated by statistically significant differences in race distribution, duration of SLE, and baseline SELENA-SLEDAI scores across the 4 clinical centers. Based on historical record review or assessment at the baseline study visit, nearly all of the patients $(96 \%)$ were antinuclear antibody positive, and $71 \%$ had arthritis. Overall, malar rash, photosensitivity, immunologic disorders, and hematologic disorders were each reported in approximately half of the patients. The incidences of arthritis, immunologic disorders, oral ulcers, and renal disorders were significantly different among the 4 study sites $(P<0.05)$. The use of cortico-

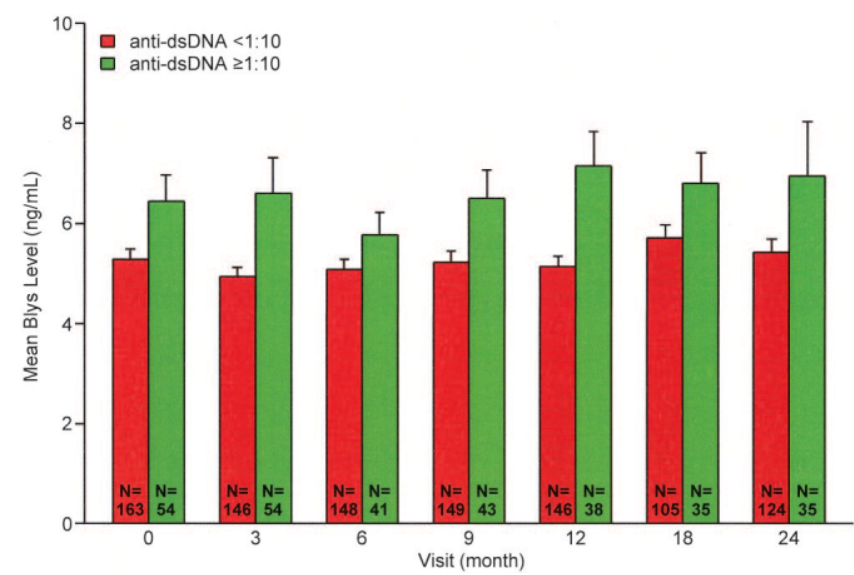

Figure 1. Levels of B lymphocyte stimulator (BLyS) in relation to anti-double-stranded DNA (anti-dsDNA) status (as determined by immunofluorescence assay) in the systemic lupus erythematosus patients at each study visit. Values are the mean and SEM. steroids and immunosuppressive drugs also differed significantly across sites (Table 1 ).

Levels of BLyS, immunoglobulin subgroups, and anti-dsDNA were assessed at baseline (Table 2). The mean baseline plasma level of BLyS in all patients combined was $5.57 \mathrm{ng} / \mathrm{ml}$ (range 1.5-19.1). At baseline, BLyS levels were positively correlated with SELENASLEDAI scores $(P=0.057)$ and $\operatorname{IgG}$ levels $(P=0.008)$. In addition, BLyS levels were significantly higher among patients with detectable anti-dsDNA antibodies $(P=$ 0.017) (Figure 1).

BLyS levels and relationship to SLE disease activity. Eighty-eight percent of the patients exhibited a $>25 \%$ change from baseline in the plasma BLyS level, on at least 1 clinical visit. Plasma BLyS levels varied over time during the 2 years of observation, with $>55 \%$ of the patients exhibiting a $>50 \%$ increase or decrease at any 1 (or more) clinical visit compared with baseline.

Association analyses (Figure 2) showed that antidsDNA levels were positively associated with BLyS concentrations $(P=0.0127)$ and with the SELENASLEDAI score $(P=0.0003)$ across all visits. BLyS levels at the current visit correlated with the SELENASLEDAI score $(P=0.0002)$, and an elevated BLyS level at the previous visit predicted anti-dsDNA positivity $(P=0.0465)$. On average, patients with BLyS levels of

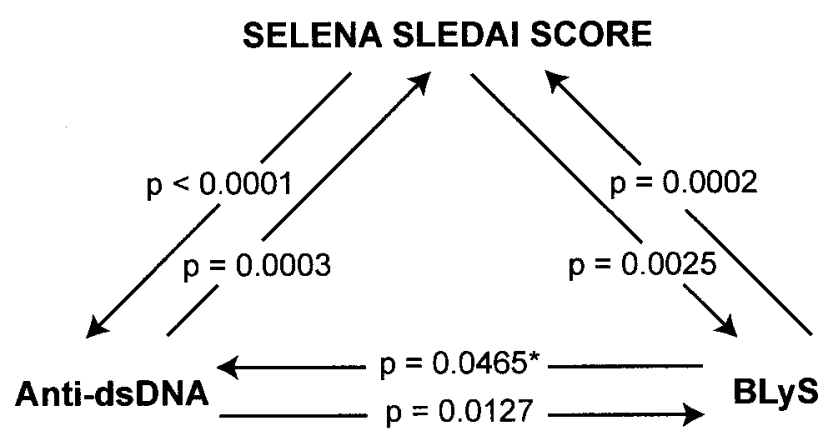

Figure 2. Associations between systemic lupus erythematosus (SLE) disease activity, presence of anti-dsDNA, and BLyS level. Score on the Safety of Estrogens in Lupus Erythematosus: National Assessment (SELENA) version of the SLE Disease Activity Index (SLEDAI) was positively associated with BLyS concentration and with the presence of anti-dsDNA. Anti-dsDNA level was positively associated with BLyS concentration and SELENA-SLEDAI score. BLyS level at the current visit associated with SELENA-SLEDAI score. BLyS level at the previous visit predicted presence of anti-dsDNA at the current visit. The direction of each arrow indicates the independent variable (predictor) and the outcome variable (e.g., the $P$ value of 0.0127 on the arrow pointing from anti-dsDNA to BLyS means that the anti-dsDNA level significantly predicts the level of BLyS). $*=$ independent variable was the BLyS level at the previous visit (all other variables refer to the current visit). See Figure 1 for other definitions. 
Table 3. Multivariate analysis of association of BLyS level with change in SELENA-SLEDAI score from previous visit*

\begin{tabular}{|c|c|c|c|}
\hline Independent variable $\dagger$ & $\begin{array}{l}\text { Relationship to } \\
\text { increase in } \\
\text { SELENA-SLEDAI }\end{array}$ & $\begin{array}{c}\text { Parameter } \\
\text { estimate }(\mathrm{SE}) \ddagger\end{array}$ & $P$ \\
\hline BLyS level at previous visit & Positive & $0.096(0.033)$ & 0.0042 \\
\hline $\begin{array}{l}\text { Change in BLyS level from previous to } \\
\text { current visit }\end{array}$ & Positive & $0.121(0.035)$ & 0.0007 \\
\hline SELENA-SLEDAI score at previous visit & Negative & $-0.639(0.028)$ & $<0.0001$ \\
\hline \multicolumn{4}{|l|}{ Race effect (nonwhite), within site } \\
\hline JHU & Negative & $-2.590(1.102)$ & 0.0196 \\
\hline USC & Negative & $-1.133(0.639)$ & 0.0774 \\
\hline UAB & Negative & $-0.141(0.353)$ & 0.6907 \\
\hline UM & Positive & $0.540(0.271)$ & 0.0478 \\
\hline Prednisone use in last 3 months & Positive & $0.405(0.200)$ & 0.0520 \\
\hline Prednisone dosage change from last visit & Negative & $-0.002(0.010)$ & 0.8168 \\
\hline Immunosuppressive drug use in last 3 months & Positive & $0.358(0.194)$ & 0.0793 \\
\hline Age & Negative & $-0.010(0.007)$ & 0.1648 \\
\hline
\end{tabular}

* Analysis was performed using a repeated-measures analysis of covariance model. See Table 1 for other definitions.

† When C4 level and presence of anti-double-stranded DNA at the current visit were added to the model, the effect of change in B lymphocyte stimulator (BLyS) level from the previous visit to the current visit and the effect of SELENA-SLEDAI score at the previous visit remained significant, and the effect of BLyS level at the previous visit was marginally significant $(P=0.0798)$.

$\$$ Parameter estimates indicate the increase in SELENA-SLEDAI score for each 1-unit increase in the given predictor (continuous variables) or the increase in SELENA-SLEDAI score associated with being in the category of the given predictor compared with not being in the category (for categorical variables) (e.g., for change in BLyS level from previous to current visit, the SELENA-SLEDAI score would increase an average of 0.121 points for each $1 \mathrm{ng} / \mathrm{ml}$ increase in the BLyS level from the previous visit to the current visit).

$>10 \mathrm{ng} / \mathrm{ml}$ had $\sim 2$-point higher SELENA-SLEDAI scores than those with BLyS levels of $\leq 10 \mathrm{ng} / \mathrm{ml}$.

A multivariate analysis was performed to investigate the relationship between the change in the SELENA-SLEDAI scores from the previous visit and BLyS levels, with adjustment for immunosuppressive drug treatment and changes in prednisone use since the last visit, SELENA-SLEDAI score at the previous visit, and baseline demographic characteristics (Table 3). The model showed that changes in the SELENA-SLEDAI score from the previous visit were significantly and positively associated with the BLyS level at the previous visit and with the change in the BLyS level from the previous to the current visit. There was a trend toward greater increases in the SELENA-SLEDAI score at the current visit among patients who had higher BLyS levels at the previous visit and among those with greater increases in BLyS levels from the previous to the current visit.

Analysis using a multivariate repeated-measures ANOVA model with adjustment for the effects of arthritis, immunologic disorders, oral ulcers, and renal disorders showed the same significant effects of both BLyS level at the previous visit $(P=0.004)$ and change in BLyS level from the previous visit to the current visit
$(P=0.0007)$. The study center effect also remained similar. The results also showed that patients with oral ulcers at the time of diagnosis were significantly more likely to have an increase in disease activity $(P=$ 0.0075).

To assess the effect of the differences in frequency of data collection between year 1 and year 2, an analysis using the multivariate repeated-measures model was performed. The results showed the same significant effects of both BLyS level at the previous visit $(P=$ 0.0034) and changes in BLyS level from the previous visit to the current visit $(P=0.0006)$. The effect of the different schedules for data collection was not statistically significant $(P=0.4619)$.

To evaluate the relationship between changes in BLyS level and development of a mild-to-moderate SLE flare (18) or improvement in SLE disease activity (19), BLyS levels were correlated with the percentage of patients who had an increase of $\geq 3$ points in the SELENA-SLEDAI score from the previous visit to the current visit, and with the percentage who had a reduction of $\geq 4$ points in the SELENA-SLEDAI score from the previous visit to the current visit. Univariate analysis showed that the increase in BLyS level from the previous visit to the current visit appeared to be associated with 
the occurrence of mild-to-moderate flares $(P=0.0512)$. In the subgroup of patients with SELENA-SLEDAI scores of $\geq 4$, improvement of $\geq 4$ points in the SELENA-SLEDAI score from the previous visit to the current visit was more likely to be observed among those who had a $\geq 1 \mathrm{ng} / \mathrm{ml}$ reduction in the BLyS level from the previous visit to the current visit with a BLyS level that was within the normal range $(1-5 \mathrm{ng} / \mathrm{ml})$ at the current visit. Twenty percent of these patients exhibited improvement of $\geq 4$ points in the SELENA-SLEDAI score, whereas only $10 \%$ of the patients with significant increases in the BLyS level (increases of $\geq 1 \mathrm{ng} / \mathrm{ml}$, from within the normal range to $>5 \mathrm{ng} / \mathrm{ml}$ ) showed improvement of $\geq 4$ points in the SELENA-SLEDAI score $(P=$ 0.0495).

\section{DISCUSSION}

Several reports have suggested a central role of BLyS in the pathogenesis of SLE $(3,5,20)$. Transgenic mice that overexpress BLyS have an increased number of B cells, enlarged lymphoid organs, and hypergammaglobulinemia, and express symptoms that resemble SLE $(5,20)$. In addition, the constitutive overexpression of BLyS in these animals is associated with antinuclear and anti-dsDNA antibodies. Elevated levels of BLyS have been found in other murine models of SLE, including

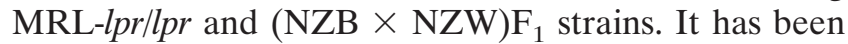
shown that treatment of mice overexpressing BLyS with a BLyS receptor fusion protein ameliorates the progression of SLE and improves survival (3). In SLE patients with B cell depletion, BLyS levels increase until B cell repopulation occurs (11).

Given the apparent link between BLyS and autoimmune disease pathogenesis in animal studies, in this prospective study we tracked BLyS levels, clinical disease activity, and biologic markers, and accounted for changes in therapy. Our study cohort should be broadly generalizable, in that it incorporates observations at 4 clinical centers with differences in patient ethnicity and medications. BLyS levels predicted increases in SLE disease activity across all study sites, supporting the notion that elevated BLyS has a role as a biomarker for current and/or future SLE disease activity. This observation was consistent when differences in disease manifestations, immunosuppressive drug treatment, study site, and race distribution were addressed in the model.

The results of this study should be contrasted with findings of past investigations. In one longitudinal cohort, SLE patients exhibited heterogeneity, with 50\% manifesting persistently or intermittently elevated plasma BLyS levels and $61 \%$ manifesting persistently or intermittently elevated blood BLyS messenger RNA (mRNA) phenotypes (17). In another study, changes in SLEDAI scores were more closely associated with changes in BLyS mRNA levels than with changes in BLyS protein levels (21).

It is important to note that the findings in the present study are based on measurement of BLyS levels in plasma. Glomerulonephritis may increase BLyS excretion in the urine, thereby resulting in lower plasma BLyS levels when renal disease contributes to the SELENA-SLEDAI score. Future analyses of urine BLyS protein and local production of BLyS (e.g., in lymphatic tissues and joints) may prove to be highly informative.

The apparent role of increased BLyS in SLE disease activity suggests that an anti-BLyS therapeutic approach may prove useful in controlling disease activity and progression. If disease activity is driven in part by a failure of dysregulated B cells to undergo apoptosis, targeting of BLyS signaling may afford the opportunity to specifically reduce pathogenic $\mathrm{B}$ cell responses while maintaining sufficient $\mathrm{B}$ cell activity to preserve normal immune function (22).

Multivariate analyses in this observational study have shown that use of immunosuppressive therapy is positively associated with disease activity and with BLyS level at the current and previous visits. Decreases in BLyS levels were associated with improvement in SLE disease activity (19), whereas increases in BLyS levels were associated with worsening of SLE disease activity indicative of mild-to-moderate flare (18). BLyS concentrations appear to be associated with current and future disease activity in patients with SLE, as measured by the SELENA-SLEDAI, and this finding supports the rationale for investigation of BLyS antagonism as a potential therapeutic approach in SLE.

\section{AUTHOR CONTRIBUTIONS}

Dr. Petri had full access to all of the data in the study and takes responsibility for the integrity of the data and the accuracy of the data analysis.

Study design. Petri, Stohl, Zhong, Freimuth.

Acquisition of data. Petri, Stohl, Chatham, McCune, Ryel, Zhong, Freimuth.

Analysis and interpretation of data. Petri, Chatham, McCune, Chevrier, Recta, Zhong, Freimuth.

Manuscript preparation. Petri, Stohl, McCune, Chevrier, Recta, Zhong, Freimuth, Dr. Lilia Pineda (nonauthor; Human Genome Sciences, Vivian Fernandez (nonauthor; Human Genome Sciences). Statistical analysis. Chevrier, Recta, Zhong.

Medical monitoring. Freimuth. 


\section{REFERENCES}

1. Moore PA, Belvedere O, Orr A, Pieri K, LaFleur DW, Feng P, et al. BLyS: member of the tumor necrosis factor family and B lymphocyte stimulator. Science 1999;285:260-3.

2. Nardelli B, Belvedere O, Roschke V, Moore PA, Olsen HS, Migone TS, et al. Synthesis and release of B-lymphocyte stimulator from myeloid cells. Blood 2001;97:198-204.

3. Gross JA, Johnston J, Mudri S, Enselman R, Dillon SR, Madden $\mathrm{K}$, et al. TACI and BCMA are receptors for a TNF homologue implicated in B-cell autoimmune disease. Nature 2000;404:995-9.

4. Thompson JS, Bixler SA, Qian F, Vora K, Scott ML, Cachero TG, et al. BAFF-R, a newly identified TNF receptor that specifically interacts with BAFF. Science 2001;293:2108-11.

5. Mackay F, Woodcock SA, Lawton P, Ambrose C, Baetscher M, Schneider $\mathrm{P}$, et al. Mice transgenic for BAFF develop lymphocytic disorders along with autoimmune manifestations. J Exp Med 1999;190:1697-710.

6. Litinskiy MB, Nardelli B, Hilbert DM, He B, Schaffer A, Casali P, et al. DCs induce CD40-independent immunoglobulin class switching through BLyS and APRIL. Nat Immunol 2002;3:822-9.

7. Dillon SR, Gross JA, Ansell SM, Novak AJ. An APRIL to remember: novel TNF ligands as therapeutic targets [review]. Nat Rev Drug Discov 2006;5:235-46.

8. Cheema GS, Roschke V, Hilbert DM, Stohl W. Elevated serum B lymphocyte stimulator levels in patients with systemic immune-based rheumatic diseases. Arthritis Rheum 2001;44: $1313-9$.

9. Zhang J, Roschke V, Baker KP, Wang Z, Alarcon GS, Fessler BJ, et al. Cutting edge: a role for B lymphocyte stimulator in systemic lupus erythematosus. J Immunol 2001;166:6-10.

10. Mariette X, Ronx S, Zhang J, Bengoufa D, Lavie F, Zhou T, et al. The level of BLyS (BAFF) correlates with the titre of autoantibodies in human Sjögren's syndrome. Ann Rheum Dis 2003;62: $168-71$.

11. Vallerskog T, Heimburger M, Gunnarsson I, Zhou W, WahrenHerlenius M, Trollmo C, et al. Differential effects on BAFF and APRIL levels in rituximab-treated patients with systemic lupus erythematosus and rheumatoid arthritis. Arthritis Res Ther 2006; 8:R167.

12. Cambridge G, Stohl W, Leandro MJ, Migone TS, Hilbert DM,
Edwards JC. Circulating levels of B lymphocyte stimulator in patients with rheumatoid arthritis following rituximab treatment: relationships with $\mathrm{B}$ cell depletion, circulating antibodies, and clinical relapse. Arthritis Rheum 2006;54:723-32.

13. Tan EM, Cohen AS, Fries JF, Masi AT, McShane DJ, Rothfield $\mathrm{NF}$, et al. The 1982 revised criteria for the classification of systemic lupus erythematosus. Arthritis Rheum 1982;25:1271-7.

14. Hochberg MC, for the Diagnostic and Therapeutic Criteria Committee of the American College of Rheumatology. Updating the American College of Rheumatology revised criteria for the classification of systemic lupus erythematosus [letter]. Arthritis Rheum 1997;40:1725.

15. Bombardier C, Gladman DD, Urowitz MB, Caron D, Chang CH, and the Committee on Prognosis Studies in SLE. Derivation of the SLEDAI: a disease activity index for lupus patients. Arthritis Rheum 1992;35:630-40.

16. Petri M, Kim MY, Kalunian KC, Grossman J, Hahn BH, Sammaritano LR, et al. Combined oral contraceptives in women with systemic lupus erythematosus. N Engl J Med 2005;353:2550-8.

17. Stohl W, Metyas S, Tan SM, Cheema GS, Oamar B, Xu D, et al. B lymphocyte stimulator overexpression in patients with systemic lupus erythematosus: longitudinal observations. Arthritis Rheum 2003;48:3475-86.

18. Petri M, Buyon J, Kim MY. Classification and definition of major flares in SLE clinical trials. Lupus 1999;8:685-91.

19. Gladman DD, Urowitz MB, Kagal A, Hallett D. Accurately describing changes in disease activity in systemic lupus erythematosus. J Rheumatol 2000;27:377-9.

20. Khare SD, Sarosi I, Xia XZ, McCabe S, Miner K, Solovyev I, et al. Severe B cell hyperplasia and autoimmune disease in TALL-1 transgenic mice. Proc Natl Acad Sci U S A 2000;97:3370-5.

21. Collins CE, Gavin AL, Migone TS, Hilbert DM, Nemazee D, Stohl W. B lymphocyte stimulator (BLyS) isoforms in systemic lupus erythematosus: disease activity correlates better with blood leukocyte BLyS mRNA levels than with plasma BLyS protein levels. Arthritis Res Ther 2006;8:R6.

22. Crowley JE, Treml LS, Stadanlick JE, Carpenter E, Cancro MP. Homeostatic niche specification among naive and activated $\mathrm{B}$ cells: a growing role for the BLyS family of receptors and ligands. Semin Immunol 2005;17:193-9. 